\title{
Research on multifunctional beds for behavioral disorders in the elderly
}

\author{
Zeng $\mathrm{Xi}^{1}$, Yi Mengdi ${ }^{1 *}$ \\ ${ }^{1}$ Art Design, Wuhan Institute Of Technology, Wuhan HuBei,China
}

\begin{abstract}
Objective Through the redesign of hospital beds, we seek better ways for elderly patients to encounter various problems due to physical mobility disorder, and to solve elderly patients, especially elderly patients with severe physical activities, in medical treatment and daily care. Methods Taking the treatment and rehabilitation needs of elderly patients as the design direction and ergonomics as the design standard, the observation method, investigation method and scenario analysis method are used to analyze the active and passive migration of elderly patients in the medical environment. The behavioral needs and behavioral state were recorded, evaluated and analyzed, and the actual problems and causes of the elderly patients due to physical movement were analyzed with the purpose and path of the behavior as clues.Conclusion Through the redesign of hospital beds, we can solve all kinds of problems that elderly patients encounter because of their body movement disorder in the process of seeking medical treatment, avoid secondary injury caused by inappropriate active or passive movement behavior of elderly patients, improve the safety and comfort of elderly patients in the process of seeking medical treatment and rehabilitation, and improve hospital doctors at the same time.
\end{abstract}

\section{Introduction}

According to statistics, since China entered the aging society in 1999 , China has become the country with the largest elderly population in the world. Among them, the prevalence of cardiovascular and cerebrovascular diseases increased significantly, and the elderly suffered frequent strokes. Because of these reasons, the number of the elderly who suffered from lower limb paralysis and illness in bed increased continuously. Therefore, the medical care of elderly patients is increasingly prominent, especially in the process of medical treatment and rehabilitation, the body of a variety of mobility encountered huge obstacles. How to overcome the frequent mobility obstacles faced by elderly patients (especially elderly patients with severe physical activity limitations) when seeking medical treatment and nursing care? The most urgent requirement for medical product design is to avoid secondary injuries caused by various shifting behaviors and improve the safety and comfort of elderly patients in the process of medical treatment and rehabilitation, at the same time improved the medical efficiency of the hospital.

\section{Analysis of medical treatment and rehabilitation needs of elderly patients}

\subsection{Status of migration behavior of elderly patients}

(1) Physical characteristics of elderly patients

Due to the deterioration of human physiology, the flexibility of the elderly limbs is gradually reduced, and various degrees of inconvenience are encountered. For elderly patients with cardiovascular disease, stroke, hemiebere hemorrhage, cerebral infarction, Guillain-Barre syndrome, etc., their limbs, waist and abdomen are difficult to move independently, this has led to a significant limitation on the basic activities of elderly patients. In order to cope with various examinations, treatments and rehabilitation measures for hospitals, frequent physical movement and transposition processes are indispensable, and a slight carelessness will most likely lead to secondary injuries in elderly patients.

(2) Hospital daily medical environment

The daily medical environment faced by elderly patients mainly includes:

1、 The environment is clean and sanitary, but there are many pathogens;

2、 There are many people in the environment, there are fixed populations associated with individual users, and a small number of non-fixed people; 
3、 The environment belongs to the public space, the space is large, convenient for wheelchairs or beds to move;

4、 The personal space is relatively small;

5、 There are professional doctor advice and nurse care, to facilitate the doctor to understand the recovery of the elderly patients body.

In summary, there are many pathogens, many people, large space and strong professional care.

In hospitals, the most frequently used and longestlasting medical device for elderly patients is the hospital bed. Whether it is a variety of examinations or routine care, elderly patients need to move from the ward's bed to the hospital's various functions or areas of activity. From the network data and field research, the migration of elderly patients in the above process is assisted by the patient's family or medical staff with the help of other equipment tools (such as wheelchairs, stretchers, operating beds, etc. ).

\subsection{Analysis of the needs of body movement in elderly patients}

Different from ordinary patients, elderly patients are affected by the physical condition and the affected parts caused by various conditions. Any form of active or passive movement will cause pain and infection to the injured part to a certain extent. For elderly patients with cardiovascular and cerebrovascular disease in the early stages of the disease, even life-threatening accidents may occur. For the analysis of the needs of migration behavior of elderly patients.

\section{Market research and design positioning analysis}

\subsection{Analysis of existing products}

At present, the main means of physical displacement of elderly patients include two types. One is that the medical staff or family members move to the destination by means of helping, and no auxiliary equipment is used in the process. Second, the medical staff or family members help the patient. After reaching the assistive device s, the elderly patient walks to his destination again.
TABLE I. Comparative Analysis of Domestic and Foreign Similar Products

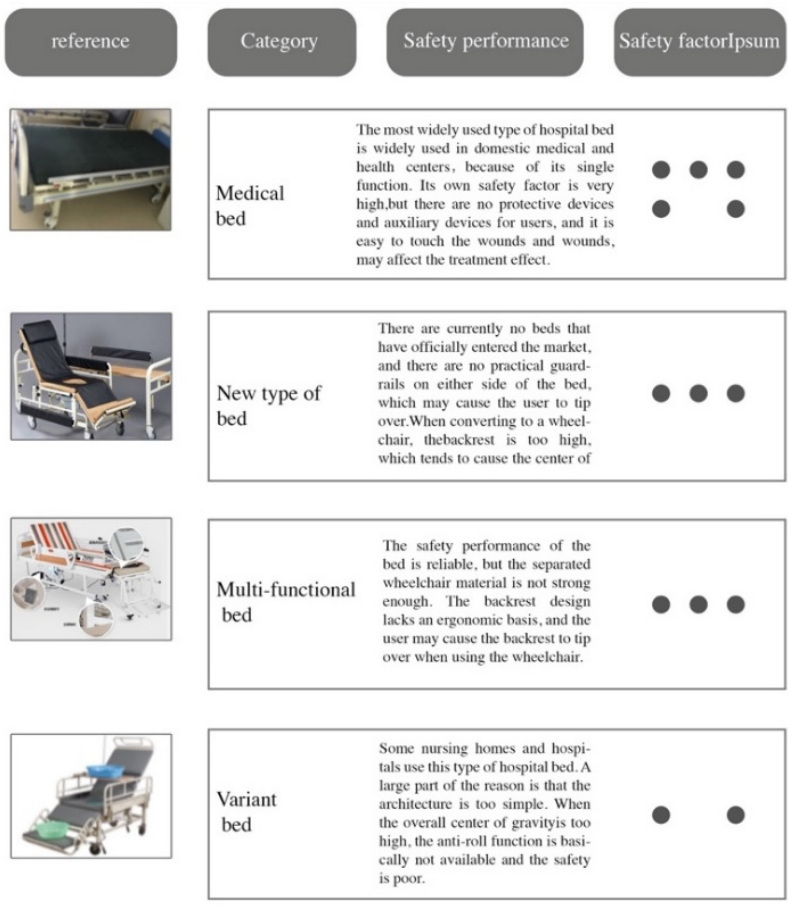

\subsection{Design Needs Positioning}

The physical disability behavior disorder faced by elderly patients is as follows:

(1) Elderly patients's can't sit and stand easily;

(2) Elderly patients's lower limbs are unable to move freely;

(3) It is difficult to keep the elderly patient's body and wound surface relatively stable when moving from the bed to the operating table ( or other special platform for treatment recovery) during surgery, thus affecting the recovery of the wound;

(4)Elderly patients with special medical conditions ( such as cardiovascular and cerebrovascular diseases, fractures, broken bones, etc. ) have deteriorated due to the slight vibration caused by the movement of the body.

When elderly patients faced with various treatment examinations in hospitals, the majority of elderly patients move in the overall movement of the beds, or they are lifted to a wheelchair or stretcher according to the doctor's advice, and then sent to the destination. In this process, various accidents and different intensity of vibrations cause different degrees of damage to elderly patients, especially in the process of artificially lifting the patientaway from the accident. For these reasons, the redesign of hospital beds is as follows:

(1) The design should quickly carry out structural deformation transformation under the premise that the elderly patients do not leave the hospital bed. And create new functions such as wheelchairs and stretchers to meet different movements.

(2) The design should meet the space constraints of various use environments to ensure smooth use. 
(3) The design should have a reasonable humanmachine structure to ensure the safety and comfort of elderly patients during use.

\section{Design scheme}

\subsection{Design sketch ( function module and structure planning)}

The bed is the most important medical device used by elderly patients. It is mainly used in various functional spaces inside the hospital. In other words, it is to go back and forth between various environments. Therefore, a folding wheelchair part that can be pulled out is designed to avoid the migration of elderly patients. Help them move safely between environments, and there must be a design that translates into a stretcher bed to cope with the moving demand for a surgical treatment that may occur.

\subsection{Solution deepening (structural improvement and material details)}

A three-dimensional model based on the sketch is shown in Figures 3, 4, 5, 6. Based on the concept of sketches and the basic care requirements of elderly patients, the threedimensional model adds to the structure of conventional beds such as turning over, helping the stool, lifting the legs and bending the legs.

The design is divided into three modes, conventional bed mode, wheelchair mode and stretcher mode. The hydraulic support rod structure is adopted in the design function mutual conversion and the lifting of the leg and the stretching leg to ensure the simple and easy operation of the conversion process; since the height of the stretcher is inconvenient to implement, it is possible to provide an upwardly extendable part on the handle to facilitate the promotion of medical personnel; In the detail part, there are velcro on the four corners of the bed to facilitate the laying of the sheets; when the wheelchair parts return to the bed, they are pushed back to the bed along the specific track of the bed.

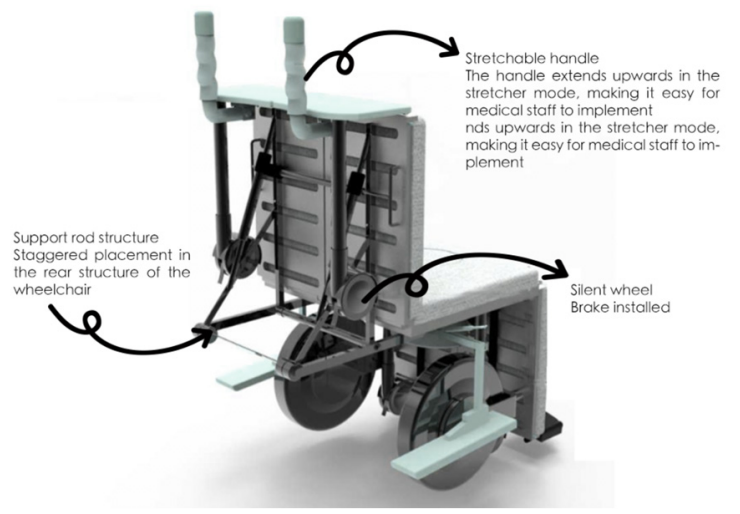

Figure 1.

Initial design scheme

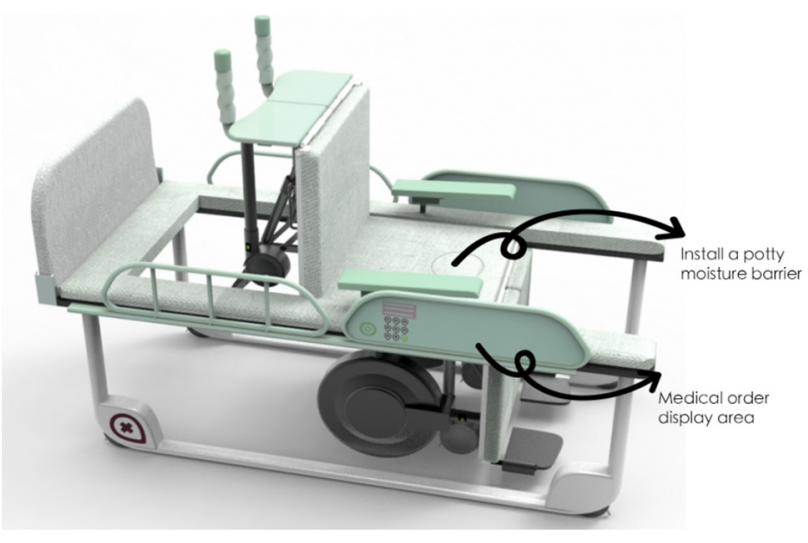

Figure 2. Initial design scheme

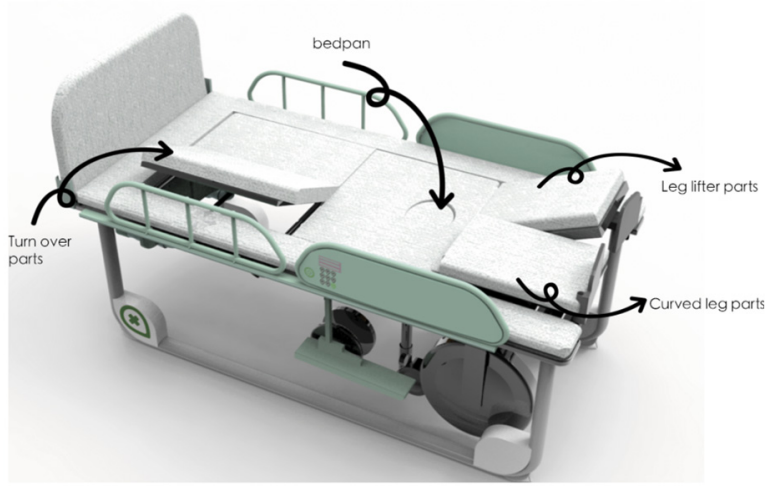

Figure 3. Initial design scheme.

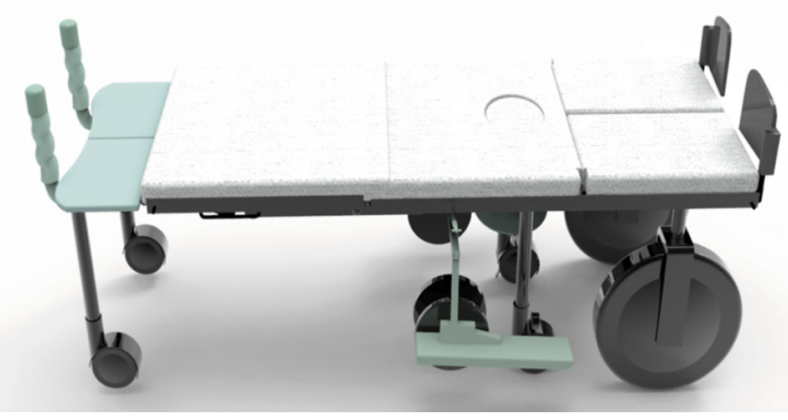

Figure 4. Initial design scheme

\section{Research on ergonomic design}

The initial scheme basically meets the needs of the original design concept of bed movement, but from the perspective of Man-machine comfort and safety, the angle, material and other factors in the process of bed conversion have not been comprehensively considered, so further improvement of the design plan is needed.

\subsection{Analysis of the comfort of the ergonomics of the bed}

There are many considerations in the ergonomic comfort of the hospital bed. On the one hand, the pressure distribution of the elderly body on the bed directly affects the comfort of the elderly. The pressure distribution of the ischial bone in the sitting and lying postures is greatest, then gradually decreases to the surrounding, and is minimal 
at the thigh. Therefore, in consideration of the stability of the pressure distribution in the design, the cushions with different degrees of hardness and hardness are installed to avoid the sudden change of pressure cause sitting instability. On the other hand, the angle at which the mechanical components of the bed are adjusted can also affect the comfort of the human joints associated with lower limb movements. Therefore, the angle of the mechanical parts of the bed should take into account the joint angle and spine curve of the elderly patients in different postures. The comfort adjustment range of the joint in different postures is determined by the actual data in Table 3.

TABLE II. Adjustment Range of Comfortable Posture of Main Joints of Human Body

\begin{tabular}{c|c|c|c}
\hline \hline $\begin{array}{c}\text { Joint } \\
\text { name }\end{array}$ & $\begin{array}{c}\text { Type of body } \\
\text { movement }\end{array}$ & $\begin{array}{c}\text { Limit angle } \\
\text { (average } \\
\text { value })\end{array}$ & $\begin{array}{c}\text { Body } \\
\text { motion } \\
\text { range }\end{array}$ \\
\hline $\begin{array}{c}\text { Thoracic } \\
\text { spine and } \\
\begin{array}{c}\text { Lumbar } \\
\text { spine }\end{array}\end{array}$ & Bend/stretch & $+80,-30$ & 110 \\
\hline & Lateral flexion & $+35,-35$ & 70 \\
\hline hip joint & Rotate & $+45,-45$ & 90 \\
\hline & Rotate & $+120,-10$ & 130 \\
\hline knee joint & Bend to stretch & $+135,0$ & 135 \\
\hline \hline
\end{tabular}

\subsection{Ergonomic comfort analysis of wheelchair parts}

\section{(1) sitting posture of static}

There is a certain contradiction between the cushion material of the traditional wheelchair and the physiological structure of the elderly patient. The pressure on the ischial tuberosity is too large, exceeding the pressure at the end of the capillaries, which can easily lead to discomfort such as ischemia and pressure sores. Elderly patients sitting in a traditional wheelchair can easily feel discomfort and fatigue. Taking into account the pressure distribution in the sitting and lying positions mentioned above, the redesigned wheelchair chose a cushion of moderate hardness to ensure wheelchair comfort.

(2) sitting posture of dynamic

In the sitting position, the main structure supporting the human body is the spine, pelvis, and legs. For elderly patients with lumbar disease, the lumbar force will gradually become smaller, and the structure supporting the weight of the human body will be transferred to the vertebrae.

Therefore, the inclination of the wheelchair backrest is slightly larger than that of the general seat, causing the force of the spine to shift upward, and the lumbar vertebrae and the backrest will bear the weight of the upper body of the elderly patient.

At the same time, the angle of inclination of the backrest should not be too large, and the optimum angle is 95-105. For the long-term sitting posture survey of the elderly, the sitting posture behavior map, as shown in Figure 7, records the changing posture, time, duration, etc. of the elderly.

According to the results of the analysis, the most frequent posture of the elderly patients is: the body leans backwards, the legs extend forward, and the buttocks are located at the trailing edge of the seat. This gives us a design direction for the backrest design.

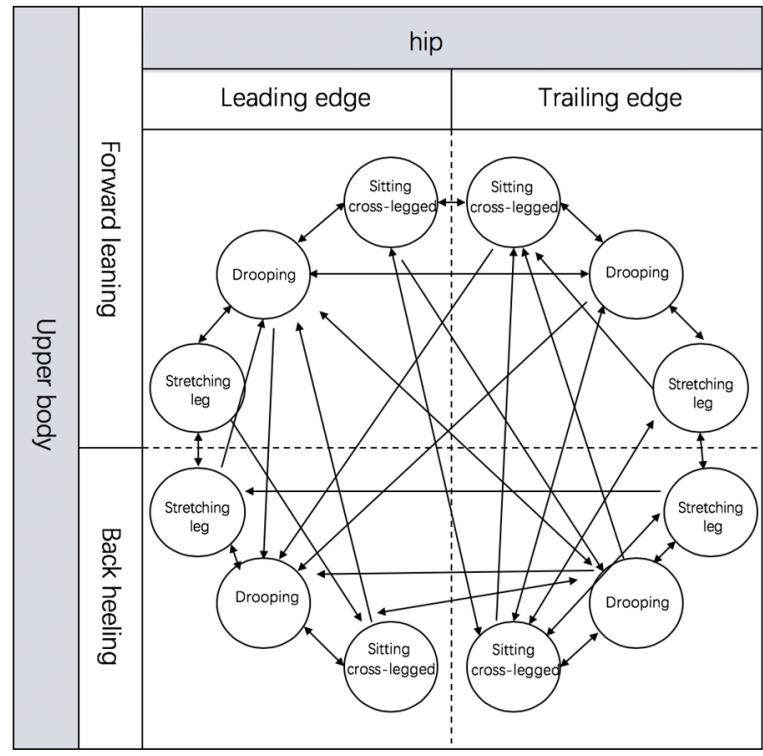

Figure 5. Sitting Behavior Transform Diagram

\section{Final design plan}

After the above ergonomic comfort analysis, the design scheme is improved. The specific scheme is shown in Figure 8, 9. On the one hand, on the basis of the original functional structure, through the dynamic sitting position under the degree of force, changed the wheelchair mode of the starting angle, the material of the cushion part, as well as the back of the bed body designed into a more comfortable semi-enclosed structure; on the other hand, in order to enhance the comfort of the use of elderly patients, the addition of integrated night lights to facilitate the use of toilets in elderly patients at night and structural components to assist elderly patients to move the head.

\section{7 conclusion}

The purpose of this study is to find a better solution to the problem of physical mobility disorders experienced by elderly patients, especially those with severe limitations in physical activity, through the re-design of hospital beds. Through the preliminary research, this study analyzes the common characteristics of the physiological and psychological conditions of elderly patients. In view of these common features and needs, this design a combintion beds, wheelchairs and stretchers to provide the necessary functional requirements while facilitating the activities of elderly patients. 
Providing better assistive devices for elderly patients is an important means to improve medical efficiency and a sign of the progress of the development of society. Over time, design systems for aging will become more and more sophisticated. In this study, the goal of this study is to overcome the physical movement disorder of elderly patients when they seek medical treatment, and we hope to solve the problems of the aging society through design, and give some suggestions on the design direction of aging in the future.

\section{References}

1. Zhou Zhanchao. Study on the Aging of Population in China [J] Comparison of Economic and Social Systems, 2007.1, 121-125.

2. Qian Wax. Research on General Design and Its Application in Old People's Furniture [D]. Nanjing Forestry University, 2010.

3. Li Jingjing. Research on Product Design and Development Trend of the Elderly [J]. Forum on Industry and Technology, 2011, 10 (08), 131-132.

4. Chen Yanxiang. Research on Vertebral Disease Early Warning Analysis [D]. Xiangjing Institute of Physical Education, 2008.

5. Wang Wenjing, Wei Liyan. Research on the Origin of Design in Human Self-conscious Behavior [J]. Science and Technology Innovation Report, 2011 (05), 22.

6. Hou Geying. Home, the place where the elderly live spiritually [J]. Meiji Times (Part I), 2014 (01): 68-70.

7. Sun Ping. Research on the Design of Urban Residential Environment for the Elderly [D]. Shenyang Architectural University, 2013.

8. Wang Xianfang, Wang Shuyang, Wang Xiaodong. Discussion on furniture design for the elderly [J]. Forestry machinery and woodworking equipment, 2006.10, 34-35.

9. Chen Yinhui. Research on Life Behavior and Furniture Design of the Elderly [D]. Nanjing Forestry University, 2008.

10. Zhang Xiaofan. Furniture Design for the Elderly [J]. Value Engineering, 2012, 31 (18): 302-303 